\title{
LA PROMOCIÓN DE LA SALUD EN EL LUGAR DE TRABAJO, UNA ALTERNATIVA PARA LOS PERUANOS
}

\author{
Raúl Gomero-Cuadra1,a, José Francia-Romero ${ }^{2, a}$
}

\begin{abstract}
RESUMEN
Las normativas de la salud en el trabajo han generado expectativas en el rol de las autoridades sanitarias en Perú. Es probable que el cuidado de la salud, basado en el tratamiento de agentes biológicos, resulte de la incomprensión de la promoción de la salud en los lugares de trabajo (PST). La PST aún es un concepto nuevo que requiere ser profundizado, sin embargo, se espera que propicie programas de salud y seguridad bien administrados, reducción del ausentismo laboral, incremento de la productividad, reducción de los costos en cuidados en la salud, reducción en problemas jurídicos y ambientes de trabajo seguros y saludables. La Organización Mundial de la Salud ratificó, en su Plan sobre la Salud de los Trabajadores, la necesidad de promover la salud, el bienestar y el trabajo saludable en el lugar de trabajo, además de promover los factores protectores frente a las enfermedades no transmisibles y ampliar el acceso a los lugares de protección de riesgos.
\end{abstract}

Palabras clave: Promoción de la salud; Salud ocupacional; Salud pública (fuente: DeCS BIREME)

\section{WORKPLACE HEALTH PROMOTION, AN ALTERNATIVE FOR PERUVIANS}

\begin{abstract}
Regulations regarding health at the workplace have generated expectations in the role played by sanitary authorities in Peru. Healthcare based on the treatment with biological agents may be a result of the lack of understanding of workplace health promotion (WHP). WHP is still a new concept that requires deepening; nevertheless, it is expected to give way to properly managed health and safety programs, reduced absenteeism, increased productivity, reduced healthcare costs, reduced legal problems, and safe and healthy work environments. The World Health Organization ratified, in its Workers Health Plan, the need to promote health, well-being, and healthy work at the workplace, in addition to promoting the protective factors against noncommunicable diseases and to extend access to risk protection.
\end{abstract}

Keywords: Health promotion; Occupational health; Public health (source: MeSH NLM)

\section{INTRODUCCIÓN}

En el año 2013 fue promulgada la Política Nacional de Seguridad y Salud en el Trabajo, con la finalidad de establecer los ejes de acción y los objetivos para el desarrollo de una cultura de prevención de riesgos laborales en el Perú, acorde con la Ley y el Reglamento de Seguridad y Salud en el Trabajo de los años 2011 y 2012, respectivamente. El Estado, los empleadores, los trabajadores, así como los profesionales de las diferentes disciplinas de la seguridad y salud realizan esfuerzos por ponerla en práctica, probablemente con logros, errores y múltiples oportunidades de mejora. En este escenario, la creación de entornos de trabajo seguros y saludables, en forma participativa y colaborativa en todos los niveles de una organización, cobra significativa relevancia. Por lo que el diseño y desarrollo de metodologías adaptadas a la realidad de cada organización, con integración de la promoción de la salud a la seguridad y salud en el trabajo, resulta una alternativa viable no sólo para prevenir y proteger la salud de los trabajadores, sino también para optimizar la responsabilidad social y la productividad de las organizaciones $\mathrm{y}$, por ende, del Perú.

\footnotetext{
Sociedad de Medicina Ocupacional y Medio Ambiente. Lima, Perú

Sociedad Peruana de Salud Ocupacional. Lima, Perú

Médico especialista en Medicina Ocupacional y Medio Ambiente

Recibido: 24/04/2017 Aprobado: 31/01/2018 En línea: 05/04/2018
}

Citar como: Gomero-Cuadra R, Francia-Romero J. La promoción de la salud en el lugar de trabajo, una alternativa para los peruanos. Rev Peru Med Exp Salud Publica. 2018;35(1):139-44. doi: 10.17843/rpmesp.2018.351.3561. 
Pese a que esta tarea es tripartita, necesita de profesionales competentes que contribuyan en su efectividad. Así, el objetivo de este artículo es analizar las directrices peruanas e internacionales en relación a la promoción de la salud en el lugar de trabajo (PST), que permita ser la base de políticas nacionales coherentes entre la salud pública y la seguridad y salud en el trabajo, considerando la participación del profesional médico y su contribución a este proceso.

\section{CONTEXTO INTERNACIONAL SOBRE LA PROMOCIÓN DE LA SALUD EN EL LUGAR DE TRABAJO} 2015-2025 de la Organización Mundial de la Salud (OMS) y la Organización Internacional para el Trabajo (OIT) solicitan a sus estados miembros, entre ellos el Perú, que el lugar de trabajo sea el ámbito de desarrollo de la promoción de la salud en el siglo XXI, reconociendo que, la prevención primaria de los peligros en el trabajo, la protección y promoción de la salud; como las condiciones de empleo, y una mejor respuesta de los sistemas a la salud de los trabajadores merecen atención prioritaria dentro de las políticas de salud de los estados, las organizaciones y del personal en el lugar de trabajo, en busca de la necesidad de brindar entornos saludables ${ }^{(1,2)}$. Esta solicitud tiene como un antecedente importante la Estrategia de Promoción de la Salud en los Lugares de Trabajo de América Latina y el Caribe organizado por la Organización Panamericana de la Salud (OPS) en el año $2000^{(3,4)}$. Resulta razonable que un ambiente de trabajo seguro y saludable sea imprescindible para lograr la salud de los trabajadores, motivación laboral, satisfacción en el trabajo y calidad de vida, en suma, productividad de los estados.

El modelo de salud pública de Marc Lalonde, estableció que los determinantes ambientales explican el $25 \%$ de nuestras enfermedades ${ }^{(5,6)}$. El ambiente debe ser entendido en su componente social y no solo biológico, de esta manera, las condiciones de trabajo, las tareas repetitivas, la exposición a contaminantes en el lugar de trabajo y el comportamiento de las personas son algunos procesos fundamentales en la promoción de la salud y la prevención de enfermedades ${ }^{(6,7)}$. Hoy sabemos que la salud de un estado no sólo se mejora y se mantiene teniendo sistemas de atención de las enfermedades ${ }^{(7)}$. También conocemos que las enfermedades causadas por los estilos de vida no saludables ocasionan el elevado costo económico a los sistemas de salud, sin embargo, la evidencia nos indica que los estilos de vida resultan de los condicionantes sociales y de la propaganda comercial ${ }^{(7,8)}$, por lo tanto, se justifica la promoción de la salud en el lugar de trabajo y en el hogar, como entornos propicios para promover la salud.

En el año 2012, la OIT presentó la segunda edición del paquete de formación denominado SOLVE, basada en la experiencia recolectada desde el año 2002, con el objetivo de integrar la promoción de la salud en el lugar de trabajo en las políticas de seguridad y salud en el trabajo de las organizaciones. El contenido de SOLVE está basado en la toma de conciencia de los factores de riesgo psicosociales en el lugar de trabajo, como el estrés, el consumo excesivo de alcohol y drogas y la prevención de la violencia, la nutrición, el VIH/SIDA, el tabaco y la exposición al humo, la actividad física para la salud, el sueño saludable y el estrés económico; debido a que afectan la salud de los trabajadores y la productividad de las empresas. Las herramientas que propone para la prevención de accidentes, enfermedades y ausentismo están basadas en el ámbito de las empresas ${ }^{(9)}$. La integración de la promoción de la salud en el lugar de trabajo debe reducir la presión asistencial sobre los sistemas de salud, bienestar y seguridad social a largo plazo. Además, su integración en la gestión de seguridad y salud en el trabajo fortalecerá las prácticas seguras y saludables, contribuyendo al desarrollo de una cultura de prevención de riesgos ${ }^{(1,9)}$.

Para la Agencia Europea para la Seguridad y Salud en el Trabajo, la PST significa mucho más que un simple cumplimiento de requisitos legales en materia de seguridad y salud, significa una contribución activa de la misma organización en la mejora de la salud y el bienestar general de sus colaboradores, tomando como base sus necesidades, las realidades respecto a la organización misma del trabajo y por supuesto, la participación de cada uno de ellos en el proceso ${ }^{(10)}$. Sin embargo, la realidad nos presenta, que mucho de lo implementado en términos de seguridad y salud en el trabajo es el esfuerzo del empleador por el cumplimiento de la legislación y no necesariamente por un verdadero reconocimiento del valor que constituye el talento humano ${ }^{(11,12)}$.

El concepto de promoción de la salud viene desde la perspectiva de la salud pública ${ }^{(13)}$. La PST debe ser entendida e implementada como la promoción de la práctica de hábitos saludables, complementando las medidas de seguridad y salud en el trabajo ${ }^{(14)}$. La OIT estima que las organizaciones en diversos países de Latinoamérica ya vienen desarrollando actividades de PST sin darse cuenta. Con el envejecimiento de la fuerza laboral, el aumento de enfermedades no transmisibles que generan enfermedades crónicas y la inclusión de personas con discapacidad, las organizaciones deben manejar las situaciones particulares y contribuir a que los trabajadores sean proactivos con el cuidado de su salud, permitiendo la continuidad de sus labores, evitando la afectación de los sistemas de salud y de las economías nacionales ${ }^{(9,14)}$.

Con la firma de la Declaración de Luxemburgo en el año 1997, se brindó una de las primeras definiciones de la PST, referida como la suma de esfuerzos de los empresarios, los trabajadores y la sociedad para mejorar la salud y el 
bienestar de las personas en el lugar de trabajo, a través de la participación activa y fomentando el desarrollo individual ${ }^{(15,16)}$.

\section{CONTEXTO NACIONAL SOBRE LA PROMOCIÓN DE LA SALUD EN EL LUGAR DE TRABAJO}

En el Perú, la Dirección General de Promoción de la Salud, definió los Lineamientos de la Política de Promoción de la Salud en el año 2005, considerando intervenciones a nivel nacional, regional y local basado en cinco ejes estratégicos: desarrollar alianzas intra e intersectoriales, mejorar las condiciones del medio ambiente, promoción de la participación comunitaria, reorientación de los servicios de salud con enfoque de promoción de la salud y reorientación de la inversión hacia la promoción de la salud y el desarrollo local ${ }^{(17)}$.

Para ello, se elaboró e implementó el Modelo de Atención Integral en Salud orientado a tres dimensiones: la persona según su etapa de vida, la familia y la comunidad; definiendo el alcance de los entornos saludables o escenarios hacia las viviendas, las instituciones educativas, los municipios y los centros laborales; colocando como comportamientos saludables o ejes temáticos: la alimentación y nutrición; la higiene y el ambiente; la actividad física; la salud sexual y reproductiva; las habilidades para la vida; la seguridad vial y cultura de tránsito y la promoción de la salud mental, buen trato y cultura de paz ${ }^{(18)}$.

Además, el Modelo de Abordaje de la Promoción de la Salud del ministerio de salud peruano considera que, para lograr el bienestar y mejoramiento de salud de los trabajadores, es necesario el aumento de la productividad, la reducción de los gastos en salud y del ausentismo y el mejoramiento de la imagen de la empresa, por lo tanto, la inversión en la mejora de las condiciones de trabajo debería incrementar la productividad de las organizaciones ${ }^{(18)}$.

Sin embargo, la reglamentación posterior en materia de seguridad y salud fue promulgada principalmente por el Ministerio de Trabajo y Promoción del Empleo, y no necesariamente estuvieron orientados al modelo de la Dirección General de la Promoción de la Salud del ministerio de salud. Además, el eje temático sobre higiene y ambiente de trabajo, estuvo orientado al acceso a cantidades suficientes de agua, la disposición sanitaria de excretas y la promoción de prácticas seguras de lavado de manos ${ }^{(18)}$, mostrando claramente que el modelo no ha integrado los aspectos de seguridad y salud propios del trabajo.

\section{DISCUSIÓN Y PERSPECTIVAS}

La implementación de la promoción de la salud en el lugar de trabajo ha permitido influenciar en la salud de los individuos, sus familias y su comunidad y, también, en los indicadores de seguridad y salud de los países y organizaciones; presentando el entorno adecuado para desarrollar intervenciones de salud pública, que permitan reducir las desigualdades en salud, a través del diálogo e inclusión de las partes interesadas ${ }^{(2,3)}$. Algunos ejemplos, son la prevención de enfermedades no transmisibles en el lugar de trabajo mediante la concientización del consumo de alimentos sanos y la práctica de actividad física; la reducción de los accidentes de trabajo mediante la seguridad basada en el comportamiento; y la prevención de enfermedades transmisibles mediante los comités de control de infecciones intrahospitalarias o la inmunización ${ }^{(8,19)}$. Estas buenas experiencias resultaron de la comprensión y suma de esfuerzos de empresarios, trabajadores y la sociedad.

La construcción e implementación de políticas de salud en Perú, debe trascender de la tradicional práctica clínica asistencial, que prioriza los agentes biológicos hacia la promoción de la salud en el lugar de trabajo, que incluya a los agentes físicos, químicos, disergonómicos y psicosociales. El objetivo sigue siendo la creación de ambientes favorables para la protección de la salud de los trabajadores y sus familias. Del mismo modo, desde el enfoque de la responsabilidad social, las organizaciones deben proporcionar un ambiente de trabajo saludable y seguro.

En marzo del 2006, Instituto Nacional de Defensa de la Competencia y de la Protección de la Propiedad Intelectual (INDECOPI) creó el comité técnico ISO 26000, con la finalidad de fomentar la gestión de la responsabilidad social dentro de las organizaciones, a través del respeto de las diferencias culturales, sociales, ambientales y legales, y de las condiciones de desarrollo económico; además de dar énfasis a los resultados de desempeño y aumentar la confianza y satisfacción de los clientes y otros stakeholders de las organizaciones ${ }^{(20)}$.

Para lograr una adecuada promoción de la salud en el trabajo, se requiere de la integración de las características y condiciones personales, la identificación de los factores de riesgo ambientales, los factores organizacionales y los aspectos sociales asociados al lugar de trabajo ${ }^{(21)}$. El enfoque y el manejo son multidisciplinarios. La temprana identificación y corrección de las características y condiciones personales que pueden agravarse luego de la interacción de los factores del lugar de trabajo, se convierte en una intervención enmarcada en la prevención primaria, secundaria y terciaria. En este contexto y aunque la evidencia científica aún no ha logrado establecer los beneficios de los exámenes médicos de ingreso con la prevención del absentismo laboral ni con los accidentes de trabajo ${ }^{(1,4)}$, estos son necesarios para los programas de intervención en la PST, debido a que constituye la línea de base del individuo y de la población, permitiendo el estudio de los periodos de latencia de enfermedades transmisibles y no transmisibles. Por este motivo, mayores y mejores estudios deben ser incentivados desde el lugar de trabajo. 
Para las organizaciones, la medición de la eficacia de la PST deberá estar acompañadas por el mejoramiento de los sistemas de vigilancia y notificación de las condiciones preclínicas y clínicas de los trabajadores, a fin de identificar y controlar los riesgos laborales ${ }^{(1,2,22)}$. También deberán considerar indicadores de tipo organizacional, productividad, satisfacción, ambiente de trabajo y económicos. La falta de conocimiento y pericia del recurso humano, la escasa investigación científica y el temor a las sanciones de nuestros empleadores no permiten investigar, en forma apropiada, el origen de los problemas. Se suma, las dificultades actuales para establecer el origen laboral en el caso de las enfermedades profesionales; los periodos de latencia largos e intermitentes entre la exposición y la aparición de la enfermedad y la combinación de exposiciones a baja dosis no permiten distinguir clínicamente una enfermedad de origen laboral de una extralaboral $(3,4,23)$.

En la $67^{a}$ sesión del comité regional de la OMS para las Américas realizada en el año 2015, el Plan de Acción sobre la Salud de los Trabajadores, concluyó que existe un subregistro de enfermedades profesionales debido a la larga latencia de estas, que hace difícil su identificación; solo entre el $1 \%$ y $5 \%$ son notificadas, al parecer, por la baja cobertura de los sistemas de salud y protección social, incluido el aseguramiento de los riesgos de trabajo y a la escasez de servicios de seguridad y salud en el trabajo, entre otros ${ }^{(23)}$.

Es correcto que deben priorizarse las acciones en materia de seguridad y salud en los sectores económicos, en los cuales las condiciones son más críticas ${ }^{(24)}$, sin embargo, no justifica que los sectores no tradicionales en la gestión de la seguridad y la salud no incorporen la promoción de la salud en sus organizaciones, incluido la realización de los exámenes médicos ocupacionales, puesto que todo empleador que crea un puesto de trabajo también está generando riesgos para el bienestar de los trabajadores, por lo que está obligado a realizar su control a través de una cultura de prevención de riesgos, en pro del respeto de los Derechos Humanos ${ }^{(20)}$. El desconocimiento en materia de salud en el trabajo exige mejorar la cobertura de los servicios de salud, integrando el desarrollo de estos en las estrategias sanitarias del país, las reformas del sector y los planes de mejora del funcionamiento de los sistemas de salud.

La acción planteada es fortalecer la descentralización de la promoción de la salud, desde los establecimientos de salud y municipios hacia los centros o lugares de trabajo, con el fin de proteger a nuestros trabajadores y sus familias. Naturalmente, también se deberán asumir tareas asistenciales, de acuerdo a las necesidades de los trabajadores ${ }^{(25)}$, a nivel de atención primaria, considerando el proceso de referencia y contrarreferencia. A pesar del progreso y la globalización, la experiencia y evidencia demuestran que no hay actividad, ni puesto de trabajo que no conlleve riesgos potenciales para la salud ${ }^{(22)}$, con especial vulnerabilidad en poblaciones específicas como niños y adolescentes, mujeres, adultos mayores y personas con discapacidad ${ }^{(26)}$.

La adaptación del trabajo a los empleados significa adecuar sus capacidades, tomando en cuenta su salud mental y física. En el año 1985, la OIT, a través del convenio $N^{\circ} 161$, definió el rol de los servicios de salud en el trabajo, asignándole funciones preventivas, de asesoría y de asistencia a los empleadores y trabajadores para mantener el entorno de trabajo seguro y saludable. Por lo tanto, corresponde a los servicios de salud asesorar en la promoción de salud en el trabajo ${ }^{(27)}$. Es comprensible que un estado de salud deteriorado afectará la productividad. La presencia de enfermedades crónicas como la diabetes, la hipertensión arterial y el cáncer, la ausencia en el trabajo por motivos de salud y las discapacidades pueden complicar el sistema de salud y la economía del país.

Los servicios de salud en el trabajo deberían ser multidisciplinarios, siendo su composición de acuerdo al índole de los programas, deben estar integrados a las demás áreas de la empresa, con independencia, tanto con el empleador como con los representantes de los trabajadores, manteniendo el manejo confidencial de la salud de las personas ${ }^{(2,27)}$. En relación a la PST, y aunque reiteramos nuestro total convencimiento que es multidisciplinaria, corresponde esencialmente a los profesionales de salud tener una destacada labor en la generación de entornos laborales seguros y saludables. Para ello, deben tener competencias que les permitan desarrollar destrezas y habilidades para elegir prácticas saludables efectivas, reforzando comportamientos propios y de sus familias; implementando programas con la participación, tanto de los empresarios como de los trabajadores o sus representantes. Es decir, lograr un entorno laboral seguro y saludable, en el que las condiciones del centro de trabajo vayan dirigidas a lograr el bienestar de los trabajadores, considerando la infraestructura o ambiente físico, las relaciones individuales, la organización, la salud emocional, la promoción del bienestar familiar y social de los trabajadores a través de la protección de los riesgos, estimulando la autoestima y el control de su propia salud y ambiente laboral ${ }^{(28)}$.

El rol del profesional médico en la PST es un derecho adquirido y necesario de la evolución misma de la salud en el trabajo, como un profesional inherente de la salud ocupacional, para la implementación de la cultura de seguridad y salud en el trabajo ${ }^{(15)}$. El desarrollo en los sectores industriales, sociales, económicos, legales y éticos en los últimos años ha sido paralelo a una mayor conciencia sobre la obligación de los estados en la protección de los trabajadores. En la realidad actual de Perú, los médicos dentro de las organizaciones deben asegurar el apoyo de la gerencia, realizar la evaluación de las necesidades, 
ordenar las necesidades en función de una escala de prioridades, elaborar, facilitar y ejecutar los planes de acción y revisar y actualizar el programa de promoción y salud en el trabajo ${ }^{(29)}$. Por lo tanto, planteamos que el profesional médico es un miembro innato de los comités de seguridad y salud en el trabajo de las organizaciones. El desconocimiento de los profesionales de la salud y las deficiencias de los sistemas de información, vigilancia y registro en los países son las principales causas del subregistro ${ }^{(12,22,23)}$. Los profesionales médicos deben realizar investigaciones que permitan el desarrollo de la promoción de la salud en los lugares de trabajo en Perú.

En relación a los programas que deberán implementarse, estos tendrían que ser determinados por factores como el número de trabajadores, sus características individuales y colectivas, sus características demográficas, el tipo de trabajo que realizan y los riesgos presentes en él, la ubicación del lugar de trabajo, el tipo y calidad de los servicios de salud disponibles en la comunidad, la capacidad económica de la empresa para brindar apoyo, entre otros, propios de cada centro de trabajo ${ }^{(25)}$.
En conclusión, la PST en su carácter multidisciplinario, debe complementar las medidas de seguridad y salud de las organizaciones, y ser la base de las políticas nacionales coherentes entre la salud pública y la seguridad y salud en el trabajo, esto no sólo favorecería la salud de las personas que trabajan, sino también mejoraría la productividad de las organizaciones. El profesional médico debe participar esencialmente en este proceso junto al resto del equipo de profesionales, de manera prioritaria, activa y competente; identificando, planificando y ejecutando la estrategia y los programas necesarios; así como, asegurando la participación efectiva de los representantes de la organización y de los trabajadores, en todos sus niveles. La implementación de los programas de promoción de la salud debe ser planificada incluyendo a los trabajadores, sus familias y la comunidad.

Contribuciones de autoría: RGC tuvo la idea y redactó el manuscrito. JFR aportó y revisó en el manuscrito original. Ambos aprobaron el documento.

Fuentes de financiamiento: El manuscrito fue autofinanciado.

Conflicto de interés: Los autores del manuscrito expresan que no tienen conflicto de interés.

\section{REFERENCIAS BIBLIOGRÁFICAS}

1. World Health Organization. 60th World Health Assembly. Workers' health: global plan of action (Resolution WHA60.26) [Internet]. Geneva: WHO; 2007 [citado 21 de enero del 2017]. Disponible en: http:// apps.who.int/gb/ebwha/pdf_files/ WHASSA_WHA60-Rec1/E/reso60-en.pdf

2. International Labour Organization. 2014 Labour Overview, Latin America and the Caribbean [Internet]. Lima: ILO; 2014 [citado 21 enero del 2017]. Disponible en: http://www.ilo.org/ wcmsp5/groups/public/---americas/-ro-lima/documents/publication/ wcms_334089.pdf

3. Organización Panamericana de la Salud. Estrategia para el fortalecimiento de la promoción de la salud en los lugares de trabajo en América Latina y el Caribe [Internet]. San José: OPS; 2000 [citado 21 de enero del 2017]. Disponible en: http://www.bvsde.paho.org/bvsast/e/ fulltext/promocion/promocion.pdf

4. Organización Panamericana de la Salud, Organización Mundial de la Salud, División de Salud y Ambiente, Programa Regional de Salud de los Trabajadores. Mesa redonda. Promoción de los ambientes de trabajo saludables: una prioridad para el desarrollo humano sostenible en el siglo XXI; 2000 [citado el 21 de enero del 2017]. Disponible en: http://www. bvsde.paho.org/bvsast/e/fulltext/ relporto/relporto.html

5. Lalonde M. Social values and public health. Can J Public Health. 1974; 65:260-8.

6. Lalonde M. The physician and health promotion. Can Med Assoc J. 1977; 116:1040-45.

7. Lalonde, M. A New Perspective on the Health of Canadians: a working document. Ottawa: Minister of Supply and Services Canada; 1974.

8. Rodriguez-Guzmán J, Piedrahita $\mathrm{H}$, Muñoz J. Actualización, edición y traducción de los logros y avances del Plan regional de salud de los trabajadores de la OPS 1995-2006 (documento de trabajo). Washington, DC: OPS; 2006.

9. Organización Internacional del Trabajo. SOLVE: integrando la promoción de la salud a las políticas de SST en el lugar de trabajo: guía del formador. Ginebra: Oficina Internacional del Trabajo-OIT; 2012 [citado 04 de abril del 2017]. Disponible en: http:// www.ilo.org/wcmsp5/groups/public/--ed_protect/---protrav/---safework/ documents/instructionalmaterial/ wcms_203378.pdf

10. FACTS 94. Promoción de la salud en el lugar de trabajo para los trabajadores
[Internet]. Bilbao: Agencia Europea para la Seguridad y Salud en el Trabajo; 2010 [citado el 04 de abril del 2017]. Disponible en: https://osha. europa.eu/es/tools-and-publications/ publications/factsheets/94.

11. Muñoz Sánchez A, Castro Silva E. Promoción de la salud en los lugares de trabajo: entre ideal e irreal. Med segur trab. 2010;56(221):288-305.

12. Bazzani L, Sánchez A. Promoción de la salud en los lugares de trabajo: un camino por recorrer. Cien Saude Colet.2016;21(6):1909-20.doi: 10.1590/141381232015216.02522016.

13. Czeresnia D, Machado de Freitas C. Promoción de la salud. Buenos Aires: Lugar editorial; 2008.

14. Barrios Casas S, Paravic Klijn T. Promoción de la salud y un entorno laboral saludable. Rev Lat Am Enfermagem. 2006;14(1):136-41.

15. Muñoz Sanchez A, Castro Silva E. De la promoción de la salud a los ambientes de trabajo saludables. Salud trab (Maracay). 2010;18(2):141-52.

16. European Network For Workplace Health Promotion. The Luxembourg Declaration on Workplace Health Promotion in the European Union [internet]. Luxemburgo: ENWHP; 1997 [citado el 04 de abril del 2017]. Disponible en: http://www.enwhp.org/fileadmin/ downloads/free/Luxembourg_Declaration_June2005_final.pdf 
17. Lineamientos de Política de Promoción de la Salud. Resolución Ministerial No 111-2005/MINSA. Ministerio de Salud; (2005) [citado el 17 de enero del 2018]. Disponible en: ftp://ftp2.minsa.gob.pe/ normaslegales/pdf/RM111-2005.pdf

18. Ministerio de Salud. Documento Técnico: Modelo de Abordaje de Promoción de la Salud en el Perú. [Internet]. Lima: Ministerio de Salud; 2006 [citado el 01 de abril del 2017]. Disponible en: http:// bvs.minsa.gob.pe/local/promocion/203 prom30.pdf.

19. Soler R, Leeks K, Razi S, Hopkins D, Aten A, Chattopadhyay S, et al. A Systematic Review of Selected Interventions for Worksite Health Promotion. The Assessment of Health Risks with Feedback. Am J Prev Med. 2010;38(2 Suppl):S237-62. doi: 10.1016/j.amepre.2009.10.030.

20. Guía de Responsabilidad Social. Norma Técnica Peruana NTP-ISO 26000:2010. INACAL. (29-12-2010). [Citado el 17 de enero del 2018]. Disponible en: https://unaaa.edu.pe/ unaaa/principal/docs/carta_desarrollo_ sostenible/7Responsabilidad_Social_ Universitaria/ISO_26000.pdf

21. Muñoz Sánchez A. Promoción de la salud en los lugares de trabajo: teoría y realidad. Med Segur Trab. 2010;56(220):220-25.

22. Gomero Cuadra R, Palomino Baldeon J, Llap Yesan C. Los programas de vigilancia médica ocupacional en los centros de trabajo. Rev Med Hered. 2006;17(2): 115.
23. Organización Panamericana de la Salud, Organización Mundial de la Salud [Internet]. Washington,D.C:OMS, OPS; 2015 [citado el 17 de enero del 2018].54.o Consejo Directivo [1 pantalla]. Disponible en: http://www.paho.org/hq/index. php?option $=$ com_content $\&$ view $=$ article\&id $=11087 \% 3$ A2015-54th-directing-council $\&$ catid $=8811 \% 3 \mathrm{Adc}$ - documents\&Itemid=41537\&lang=es\#Documentosoficiales

24. Modifican el Reglamento de la Ley $N^{\circ}$ 29783, Ley de Seguridad y Salud en el Trabajo, aprobado por Decreto Supremo N ${ }^{\circ}$ 005-2012-TR. DECRETO SUPREMO No 016-2016-TR. El Peruano. (22-12-2017). [citado el 17 de enero del 2018]. Disponible en: http:// busquedas.elperuano.pe/normaslegales/ modifican-el-reglamento-de-la-leyn-29783-ley-de-seguridad-decretosupremo-n-016-2016-tr-1466666-6/

25. Rantanem J, Fedotov I. Standards, principles and approaches in occupational health services. [Internet]. ILO; 2001. [citado el 17 de enero del 2018). Disponible en: http://www.ilo.org/ wcmsp5/groups/public/---ed_protect/--protrav/---safework/documents/ publication/wcms_110439.pdf

26. Aprueban el Reglamento de Seguridad y Salud en el Trabajo de los Obreros Municipales del Perú. DECRETO SUPREMO No 017-2017-TR. El Peruano. (04-08-2017). [citado el 17 de enero del 2018]. Disponible en: http://busquedas. elperuano.pe/normaslegales/apruebanel-reglamento-de-seguridad-y-salud-enel-trabajo-de-decreto-supremo-n-0172017-tr-1551410-1/

27. Organización Internacional del Trabajo [Internet]. Ginebra: OIT; 1985. [citado el 18 de enero del 2018]. C161 - Convenio sobre los servicios de salud en el trabajo, 1985 (núm. 161) [2 pantallas]. Disponible en: http://www.lo.org/ dyn/normlex/es/f ?p=NORMLEX PUB:12100:0::NO::P12100_ILO_ CODE:C161

28. Organización Mundial de la Salud. Entornos Laborales Saludables: Fundamentos y Modelo de la OMS Contextualización, Prácticas y Literatura de Apoyo [Internet]. Ginebra: OMS; 2010 [citado el 17 enero del 2018]. Disponible en: http:// www.who.int/occupational_health/evelyn_hwp_spanish.pdf

29. Hymel P, Loeppke R, Baase C, Burton W, Hartenbaum N, Hudson $\mathrm{T}$, et al. Workplace health protection and promotion: a new pathway for a healthier-and safer-workforce. J Occup Environ Med. 2011;53(6):695-702. doi: 10.1097/ JOM.0b013e31822005d0.

Correspondencia: Raúl Jesús Gomero Cuadra Dirección: Jr. Paseo de Aguas Mz D Lote 18 Correo electrónico: raul.gomero.c@gmail.com

\title{
Ahora nuestra revista incluye:
}

\author{
$\checkmark$ Publicación anticipada \\ $\checkmark$ Compartiendo publicaciones científicas con el ciudadano \\ $\checkmark$ Videos de presentaciones conjuntas del Instituto Nacional de \\ Salud y la Academia Nacional de Medicina \\ Galería fotográfica y uideos
}

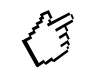

MINISTERIO DE SALUD

Instituto Nacional de Salud

Síguenos en: 